\title{
1 LABELLING HUMAN KINEMATICS DATA USING CLASSIFICATION MODELS
}

2 February $18^{\text {th }}, 2022$

3 Machine Learning Classification models can produce highly accurate sensor labels for

4 motion data captured in the motion capture studio

5

6 Yuan Shi, ${ }^{1}$ Nihir Chadderwala, ${ }^{2}$ Ujjwal Ratan ${ }^{3}$

$7 \quad{ }^{1}$ Amazon Web Services, Singapore, Singapore

$8 \quad{ }^{2}$ Amazon Web Services, Dallas, TX, USA

$9{ }^{3}$ Amazon Web Services, Seattle, WA, USA

10

11 Conflict of Interest Disclosure: None.

12

13 Correspondence Address:

14 Ujjwal Ratan

15 Amazon Web Services,

$1622057^{\text {th }}$ Avenue,

17 Seattle, WA 98121

18 Phone: (206) 615-5400, Email: ujjwalr@amazon.com

19

20

21

22 


\section{Abstract}

24 The goal of this study is to develop a classification model that can accurately and 25 efficiently label human kinematics data. Kinematics data provides information about the 26 movement of individuals by placing sensors on the human body and tracking their 27 velocity, acceleration and position in three dimensions. These data points are available in 28 C3D format that contains numerical data transformed from 3D data captured from the 29 sensors. The data points can be used to analyse movements of injured patients or patients 30 with physical disorders. To get an accurate view of the movements, the datasets generated

31 by the sensors need to be properly labelled. Due to inconsistencies in the data capture 32 process, there are instances where the markers have missing data or missing labels. The 33 missing labels are a hindrance in motion analysis as it introduces noise and produces

34 incomplete datapoints of sensor's positioning in 3 dimensional space. Labelling the data 35 manually introduces substantial effort in the analysis process. In this paper, we will 36 describe approaches to pre-process the kinematics data from its raw format and label the 37 data points with missing markers using classification models.

38 Keywords: machine learning, c3d, biomechanics, classification model 


\section{Introduction}

47 Biomechanics refer to the study of mechanical laws relating to the movement of living

48 organisms.(1) When applied to humans and combined with kinematics, it helps us capture

49 data which is used to infer a variety of human actions. For example, kinematics data is

50 used to understand human's movements in each gesture, through providing quantitative

51 data to evaluate each individual's flexibility(2). It is also used to analyse patients'

52 movements to track their recovery and assist towards rehabilitation(3). Kinematics data is

53 captured in dedicated labs setup as motion capture studios. In some cases, the sensor data

54 may not be labelled completely. This can happen for a variety of reasons like the sensor

55 not being attached securely or some sensors getting blocked from a camera from certain

56 angles. This introduces noise and missing values in the dataset and poses a challenge for

57 further analysis of this data. Capturing movements of these patients and labelling them

58 can be very tedious and an expensive process. To solve this problem, we describe a

59 method where we use the labelled data from these sensors for training a classification

60 model that can then label the unlabelled sensor records with high accuracy, thereby

61 reducing manual efforts.

62 The primary objective of this study is to use a classification model to label the sensor data

63 corresponding to the mounted location on the human subject's body. We took the records

64 with pre-labelled information and built classification models that would identify the right

65 class (sensor label) for the unlabeled dataset. In this study, we firstly clean and

66 standardize the raw C3D files through data transformation functions. We then use the

67 processed data to train 4 different machine learning models to classify the sensor data

68 points into one of the multi-class labels. Lastly, we evaluate the performance of the 
69 models to auto generate labels with test dataset. Interpretation and future work is

70 provided at the end of the paper.

\section{Method}

72 In this section, we go into the details of our method starting with describing the raw

73 dataset, data processing and feature engineering, modelling approaches including the

74 choice of algorithms and the evaluation methods.

\section{Data Description}

77 For the purposes of this study, we downloaded the raw data from CMU Graphics Lab

78 Motion Capture Database in $C 3 D$ format(4), which is a file format that has been widely

79 used in Biomechanics, Animation and Gait Analysis laboratories to record synchronized

$803 d$ kinematics data. It contains information needed to read, display, and analyse $3 d$

81 motion data and additional analog data from force plates, electromyography,

82 accelerometers, and other sensors. The dataset contains numerical data extracted from

83 sensors attached to the human body. It is composed of a list of time-series points. Each

84 point is composed of $x, y$ and $z$ co-ordinates, time of capturing, frame number and the

85 labeled location. The choice of number of sensors and locations mounted vary in each

86 setting as it subjects to the purpose of specific biomedical study. For more details, readers

87 can refer to CMU Graphics Lab Motion Capture Database (4) to visualize how the

88 sensors are mounted on the human body to capture motion data.

89

90 The $C 3 D$ dataset being captured can be converted into a standard dataframe (Table 1)

91 using the $c 3 d$ python library(5). 
92 Table 1: C3D Sample File in DataFrame

\begin{tabular}{cccccccc}
\hline time & $\mathbf{x}$ & $\mathbf{y}$ & $\mathbf{z}$ & cam & err & frame & point_label \\
\hline $\mathbf{1 . 0 1 2 5}$ & 0 & 0 & 0 & FALSE & FALSE & 1 & R_ASIS \\
$\mathbf{1 . 0 1 2 5}$ & 0 & 0 & 0 & FALSE & FALSE & 1 & L_ASIS \\
$\mathbf{1 . 0 1 2 5}$ & 0 & 0 & 0 & FALSE & FALSE & 1 & SACRUM \\
$\mathbf{1 . 0 1 2 5}$ & 0 & 0 & 0 & FALSE & FALSE & 1 & R_THIGH_1 \\
$\mathbf{1 . 0 1 2 5}$ & 0 & 0 & 0 & FALSE & FALSE & 1 & R_THIGH_2 \\
\hline
\end{tabular}

93 Note: This table captures the structure of a raw C3D file when its converted into a dataframe.

94

95

96

97 from $0, x, y$ and $z$ correspond to captured location at $x, y$ and $z$ axis respectively. cam

As shown in Table 1, a typical $C 3 D$ dataset has 8 features, correspond to time, , $x, y, z$, cam, err, frame and point_label. Here, time refers to the capture time of the point starting suggests if there is any camera observing the sensor. To ensure accuracy of captured data, it is required to ensure that at least one camera is observing a sensor at the indicated time. Otherwise, the data point is advised to be removed from the dataset. err suggests whether there is error in capturing the $3 d$ location at this frame, and frame refers to the time frame of the current position with continuous integer starting from 1. Point_labels are the labelled targets which indicate the location where the sensor is attached to.

In our experiment, we downloaded a collection of C3D files of subject \#26 provided by Qualisys illustrating human gait(6). Among all the collections, we selected all the files titled as hybrid walking motions, containing $5 \mathrm{C} 3 \mathrm{D}$ files in total.

\section{Data Processing and Feature Engineering}

Data processing is an important stage to prepare data for machine learning models. In addition, data processing gives us a chance to gain insight into the data and perform feature engineering. Using C3D python library (5), we were able to extract $x, y$ and $z$ coordinates for each frame of the motion. We followed the following data processing steps :
1) Identify all missing values and convert them to $\mathrm{NaN}$ 
113 An initial investigation of the dataset showed that all the missing values captured by

114 sensors have been recorded as 0 . As column $\mathrm{z}$ has only positive data, while both $\mathrm{x}$ and $\mathrm{y}$

115 can have negative, positive and 0 values, we used $z$ as reference to identify missing

116 values and convert all the corresponding values from all axis to $\mathrm{NaN}$ if $z=0$.

117 2) Removal of missing values.

118 Removal of missing values includes:

119 a) removal of sensors which failed to capture locations with $50 \%$ or more

120 of the total frames. After applying the filtering criteria, we were left with 19 labels

121 among the initial 25 labels in total.

b) removal of frames with consecutive missingness $>3$ as too many

123 consecutive missingness will result in bad interpretation during data processing.

124 3) Interpolation

125 Polynomial interpolation was later carried out with sensors that contain missing

126 values. We chose polynomial interpolation with 3-degree as it is simple and is

127 still able to approximate complicated curves. As showed in the equation below,

128 we refer to $P(x)$ as the polynomial function with degree of 3 .

$$
\begin{aligned}
& L_{n, j}(x)=\prod_{k \neq j} \frac{x-x_{k}}{x_{j}-x_{k}} \\
& P(x)=\sum_{j=0}^{n=3} y_{j} L_{n, j}(x)
\end{aligned}
$$




\section{3) Feature Engineering}

130 Feature engineering was conducted on the sensor data based on kinetic understanding.

131 The sensors are attached to human body, each part of human body will move according to

132 a specific trajectory, thus the spatial change of each sensor can be a good indicator of

133 where the sensor is mounted on the human body. Based on this assumption, we included

134 the following features in our training set:

135 1) Absolute location: $x, y$ and $z$ values directly generated from the device.

136 Here, $t$ refers to the time point of measurement and $x_{t}, y_{t}$ and $z_{t}$ refer to

137 the absolute position of respective axis at time $t$.

138 2) Relative location: the relative rank of each sensor at time point $t$. It is

139 calculated through ranking each point $x, y$ and $z$ values among all the $x, y$

$140 \quad$ and $z$ at each time $t$ accordingly. Let's take $x$ for example.

$$
x_{t R}=\operatorname{Rank}\left(x_{t}\right)
$$

3) Relative change (1-dimensional): the change to the current frame from previous $i$ frame(s).

$$
x_{t-R i}=x_{t}-x_{t-i}
$$

4) Relative change (3-dimenisonal): the change from previous frame to the current frame.

$$
D_{t}=\sqrt[2]{\left(x_{t_{-} A}-x_{t-1_{A}}\right)^{2}+\left(y_{t_{-} A}-y_{t-1 \_}\right)^{2}+\left(z_{t_{-} A}-z_{t-1 \_A}\right)^{2}}
$$

149 In the classification models, the raw data as absolute location (feature 1) plus time

150 is used in our MLP base and LSTM models, while raw data and enhanced features

151 1-4 are used in MLP and XGBoost classification. 
152 After feature engineering, the first 4 files are connected together as training

153 dataset and the file 5 is left as testing dataset.

$154 \quad$ Modelling Algorithms

155 Our goal of the experiment was to accurately classify the sensor data into one of

156 the 19 sensor labels in our dataset. We assumed the point_label column as the

157 target variable for this classification task. We selected multilayer perceptron

158 (MLP) base model using scikit-learn, XGBoost and Long Short-Term Memory

159 (LSTM) networks for this work.

160

161 Baseline Model with Multi-Layer Perceptron

162 We decided to use Multilayer Perceptron (MLP) as our base model (Figure 1) as they are

163 good for both classification and regression problem and can work very well with tabular

164 dataset.

165

Figure 1: Model topology of MLP baseline. In the basic model, we used raw kinematic 23 values corresponding to the 23 unique sensor labels.

169

170 MLPs are universal function approximators as shown by Cybenko's theorem(7),

171 and it is a classical neural network where we have input layer, with one or more

172 hidden layer and an output layer.

173

174

$$
=\varphi(\Sigma=1+)=(+)
$$

175 
176 Here, $w$ denotes the vector of weights, $x$ is the vector of inputs, $b$ is the bias and $\varphi$

177 refers to the non-linear activation function.

178 Training MLP involves multiple passes on the dataset while at the same time

179 adjusting weights and biases in relation to the error with the goal of reducing the

180 error. MLP model adjusts the weights and biases using a technique called

181 backpropagation. During forward pass, our input vector passes through the input

182 layer and activation function. The result is then compared with the ground truth

183 value to calculate the error using a loss function. In the backward pass, we

184 compute the gradients using stochastic gradient descent algorithm and adjust the

185 weights and biases. Weights and biases are adjusted in order to reduce the error

186 when making classification.

187 Enhanced MLP

188 On top of all the absolute features that we included in MLP baseline model, we

189 further included enhanced features as mentioned in 2.2 data processing section.

190 As evident from the diagram (Figure 2), additional features are fed into the model as

191 inputs. Features such as frame and time were removed for this model while new features

192 such as changes of position in $x, y$ and $z$ from previous time point to current time point

193 were added. Training the model using feature engineered data helped improve the model.

Figure 2: Model topology of MLP enhanced. In this enhanced model, we included

196 features as introduced in method section while retaining model structure as baseline model. 
$201 \underline{\text { XGBoost }}$

202 XGBoost was initially proposed by Chen and Guestrin in 2016(8) and it 203 implements machine learning algorithms under the Gradient Boosting 204 framework(9). Specifically, XGBoost is a decision-tree-based ensemble machine 205 learning algorithm based on optimized distributed gradient boosting library and is 206 thus highly efficient, flexible and portable. We followed the default 207 hyperparameters, except for objective being changed to multi:softmax and the 208 number_class being changed to the corresponding 19 classes in the raw C3D 209 dataset.

$210 \underline{\text { LSTM }}$

211 Long short-term memory (LSTM) is an artificial recurrent neural network (RNN) 212 architecture(10) with a LSTM unit composed of a cell, an input gate, an output gate and a 213 forget gate. For the model topology (Table-2), a sequential model which is a linear stack 214 of layers is used. the first layer is an LSTM layer with memory units and it returns 215 sequence. A dropout layer is applied to avoid overfitting of the model; after that, a dense 216 layer with activation algorithm of relu is added, followed by a dense layer with softmax 217 function for classification.

218 For the hyperparameters, we set target to maximize categorical_crossentropy,

219 with 80 epochs and batch size of 100.

220 Table 2: Model topology of LSTM

221
222
223
225
226

\begin{tabular}{|c|c|c|}
\hline Layer (type) & Output Shape & Param \# \\
\hline 1stm_2 (LSTM) & (None, 50) & 10800 \\
\hline dropout_2 (Dropout) & (None, 50) & 0 \\
\hline
\end{tabular}




dense_4 (Dense) $\quad$ (None, 19) 2451

237 Model Evaluation Approach

\section{$238 \underline{\text { F1 score }}$}

239 We are dealing with multi-class classification for a set of time-series data points and the

240 purpose is to classify each set of data into one of the classes. The total dataset is randomly

241 split into training and testing dataset with ratio of $0.8,0.2$. Evaluation is carried out on the

242 testing dataset. As the data labels are not uniformly distributed, we chose the F1 score as

243 the harmonic mean of the precision and recall.

$$
\begin{aligned}
& \text { F1 score }=2 * \frac{\text { precision } * \text { recall }}{\text { precision }+ \text { recall }} \\
& \text { accuracy }=\frac{1}{N} \sum_{k=1}^{|G|} \sum_{x: g(x, y, z)} I(g(x, y, z)=\widehat{g})
\end{aligned}
$$

244 Where precision (also called positive predictive value) is the fraction of true

245 positive samples among the predicted true samples, while recall (also known

246 as sensitivity) is the fraction of true positive samples among all the positive

247 samples.

248 Confusion matrix

249 A confusion matrix is also known as an error matrix. It is a table layout that allows 250 visualization of the performance of a supervised learning model. Each row of 251 the matrix represents the instances in an actual class while each column represents the 252 instances in a predicted class, or vice versa. 


\section{Results}

254 Both training dataset and testing dataset were applied with the same data cleaning

255 strategies with polynomial interpolation and all the feature engineering as described in the

256 methodology. After that, we built four models with MLP baseline, MLP enhanced model,

257 XGBoost enhanced model and LSTM using training dataset. The performance was

258 measured on the testing dataset.

259 Model Accuracy

260 The Table 3 below showed performance (F1 score) of the four models with the same

261 testing dataset. As shown in the table, XGBoost showed highest performance with f1

262 score at 0.94 , followed by MLP enhanced (F1 score=0.86), LSTM(F1 score=0.65) and

263 MLP baseline (F1 score=0.64).

264 Table 3: Performance comparison among 4 models

\begin{tabular}{|c|c|c|c|}
\hline Model & Algorithm & Feature & F1 score \\
\hline MLP baseline & MLP & absolute location, frame & 0.64 \\
\hline MLP enhanced & MLP & $\begin{array}{l}\text { absolute location, relative location, } \\
\text { changes }\end{array}$ & 0.86 \\
\hline XGboost enhanced & XGBoost & $\begin{array}{l}\text { absolute location, relative location, } \\
\text { changes }\end{array}$ & 0.94 \\
\hline LSTM & LSTM & absolute location & 0.66 \\
\hline
\end{tabular}

265 Note: This table captures the results of the various algorithms and models used in the evaluation with

266 their corresponding F1 scores.

267 Confusion matrix to show agreement between truth and prediction from the model

268 As XGboost showed highest performance among all the 4 models, we chose predictions

269 from XGboost and use a confusion matrix to demonstrate the agreement between true

270 labels and model output (Figure 3). As illustrated by the side bar, lighter colors refer to

271 higher number while darker colors indicate lower number. In the confusion matrix, we

272 can observe that the matrix diagonal are in lighter color, suggesting high agreement

273 between true labels and predicted labels. Nevertheless, Mislabelling was observed,

274 especially more frequent between $R \_H E E L$ and $R \_M T 5$. 
medRxiv preprint doi: https://doi.org/10.1101/2022.02.18.22271206; this version posted February 24, 2022. The copyright holder for this preprint (which was not certified by peer review) is the author/funder, who has granted medRxiv a license to display the preprint in perpetuity.

It is made available under a CC-BY 4.0 International license.

Figure 3: Confusion matrix of true labels and predicted labels from model with XGBoost.

\section{6}

277 Feature Importance

278 As XGboost showed highest accuracy among all the model, we further examined

279 the model by checking feature importance. As we can see in figure 4, it showed

280 that relative location of $y$ together with $z$ has the highest importance in the

281 modelling, followed by absolute position of $\mathrm{z}$.

282

Figure 4: Feature Importance from model with XGBoost.

283

\section{Discussion}

285 Capturing human kinematics data requires mounting sensors to pre-defined positions on

286 different parts of the subject's body, which may lead to missing labels. In current motion

287 capture pipelines, manual validation and labelling is a time consuming and labour 288 intensive postprocessing step and may become a bottleneck for downstream analysis.

289 Prior to this, Holzreiter(11) used neural network to estimate the positions of sorted 290 markers from a shuffled set through pairing up the marker locations with the shuffled set 291 using the nearest neighbour search. Meyer et al.(12) estimated the skeletal configuration 292 by least-squares optimization and exploited the skeletal model to automatically label the 293 markers. Besides, Han et al.(13) proposed auto-labelling approaches specifically for 294 labelling hands' kinematics data through keypoint regression problem solved with 295 convolutional neural networks. In addition, Saeed et al.(14) used a data-driven approach 296 for automatic labelling through permutation estimation where shuffled markers are ranked 297 based on a pre-defined order. 
298 While the previous work has no labelled data and tried to classify the kinematic data to

299 different locations according to the temporal profile, our work targets to label kinematic

300 data with missing labels. Specifically, the problem we are focusing on is the data quality

301 of the captured data with majority of the data being labelled and a few missing data points

302 due to technical errors. After effective feature engineering and modeling with 3 selected

303 model algorithms, we are able to achieve decent classification outome with F1-score over

$30490 \%$.

305 The list of features, model performance and feature importance will vary based on the

306 type of motion being captured by the sensors. In this experiment, we used the motion data

307 of a walking subject, on which over 20 sensors were mounted. Since the motion was

308 horizontal, all sensors moved by similar distance along the $x$ axis as the subject moved.

309 As a result, the relative position of $x$ did not contribute as much to differentiation of

310 sensors, as compared to $y$ and $z$ axes, because the sensors were mounted at different

311 hights on the subject's body. To apply the models to other movements, we need to retrain

312 the model with least effort since the list of features, model performance and feature

313 importance will vary based on the type of motions being captured by the sensors. We also

314 noticed that appropriate feature engineering was essential to get higher accuracy. By

315 using relative locations of the sensors along the $x, y$ and $z$ axes, we were able to improve

316 the performance of the model by over $31 \%$ compared to the baseline model that used just

317 the absolute locations of the sensors. This shows that relative location was the key factor

318 in determining the appropriate sensors. 
320 We tested different data transformation and machine learning algorithms to develop a

321 multi-class classification model that can label kinematics data with high accuracy. It was

322 also important to apply techniques on kinematics timeseries data as it helped us to

323 improve the model. Calculating the 1-dimensional and 3-dimensional relative change to

324 the frames helped with creation of new features. These techniques improved the model

325 performance considerably. XGBoost model gave the best performance compared to the

326 neural architecture models. The models can be used to accurately label the three-

327 dimensional motion data which can provide insights into movements of a patient with

328 injury or a patient with disability. Analyzing these movements can further help in either

329 creating a recovery plan or an exoskeleton that can aid in recovery.

\section{$331 \quad$ Reference}

332 1. Hatze H. The meaning of the term biomechanics. J Biomech. 1974;7(12):189-190.

333 2. An K, EY C. Kinematic analysis of human movement. Ann Biomed Eng. 334 1984;12(6):585-97. doi: 10.1007/BF02371451. PMID: 6534225. Ann Biomed Eng.

$3351984 ; 12(6): 585-97$.

336 3. Bahl JS, Nelson MJ, Taylor M, Solomon LB, Arnold JB, Thewlis D.

337 Biomechanical changes and recovery of gait function after total hip arthroplasty for 338 osteoarthritis: a systematic review and meta-analysis. Osteoarthr Cartil. $3392018 ; 26(7): 847-63$.

340 4. CMU Graphics Lab Motion Capture Database [Internet]. Available from: 341 http://mocap.cs.cmu.edu/faqs.php

342 5. Lab TEC. c3d 0.3.0 [Internet]. Python Library. Available from: 
343 https://pypi.org/project/c3d/

344 6. C3D.ORG. No Title. p. https://www.c3d.org/sampledata.html.

345 7. Cybenko G. Approximation by superpositions of a sigmoidal function. Math

346 Control Signals, Syst. 1989;2(4):303-314.

347 8. Chen T, Guestrin C. XGBoost: A scalable tree boosting system. In: Proceedings of 348 the ACM SIGKDD International Conference on Knowledge Discovery and Data $349 \quad$ Mining. 2016.

350 9. Friedman JH. Greedy Function Approximation: A Gradient Boosting Machine. $351 \quad 1999$

352 10. Schmidhuber SHJ. Long short-term memory. Neural Comput. 1997;9(8):17353531780.

354 11. Holzreiter S. Autolabeling 3D tracks using neural networks. Clin Biomech. $355 \quad 2005 ; 20(1): 1-8$.

356 12. Meyer J, Kuderer M, Müller J, Burgard W. Online marker labeling for fully 357 automatic skeleton tracking in optical motion capture. 2014 IEEE Int Conf Robot $358 \quad$ Autom. 2014;

359 13. HAN S, LIU B, WANG R, Ye Y, TWIGG CD, KIN K. Online Optical Marker360 based Hand Tracking with Deep Labels. ACM Trans Graph. 2018;37(4).

361 14. Ghorbani S, Etemad A, Troje N. Auto-labelling of Markers in Optical Motion 362 Capture by Permutation Learning. Comput Vis Pattern Recognit. 2019;167-78. 


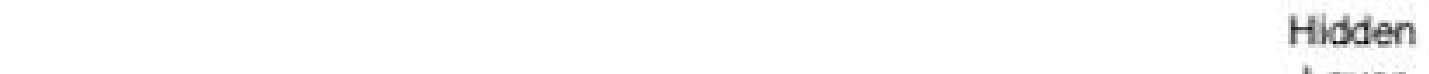

Output

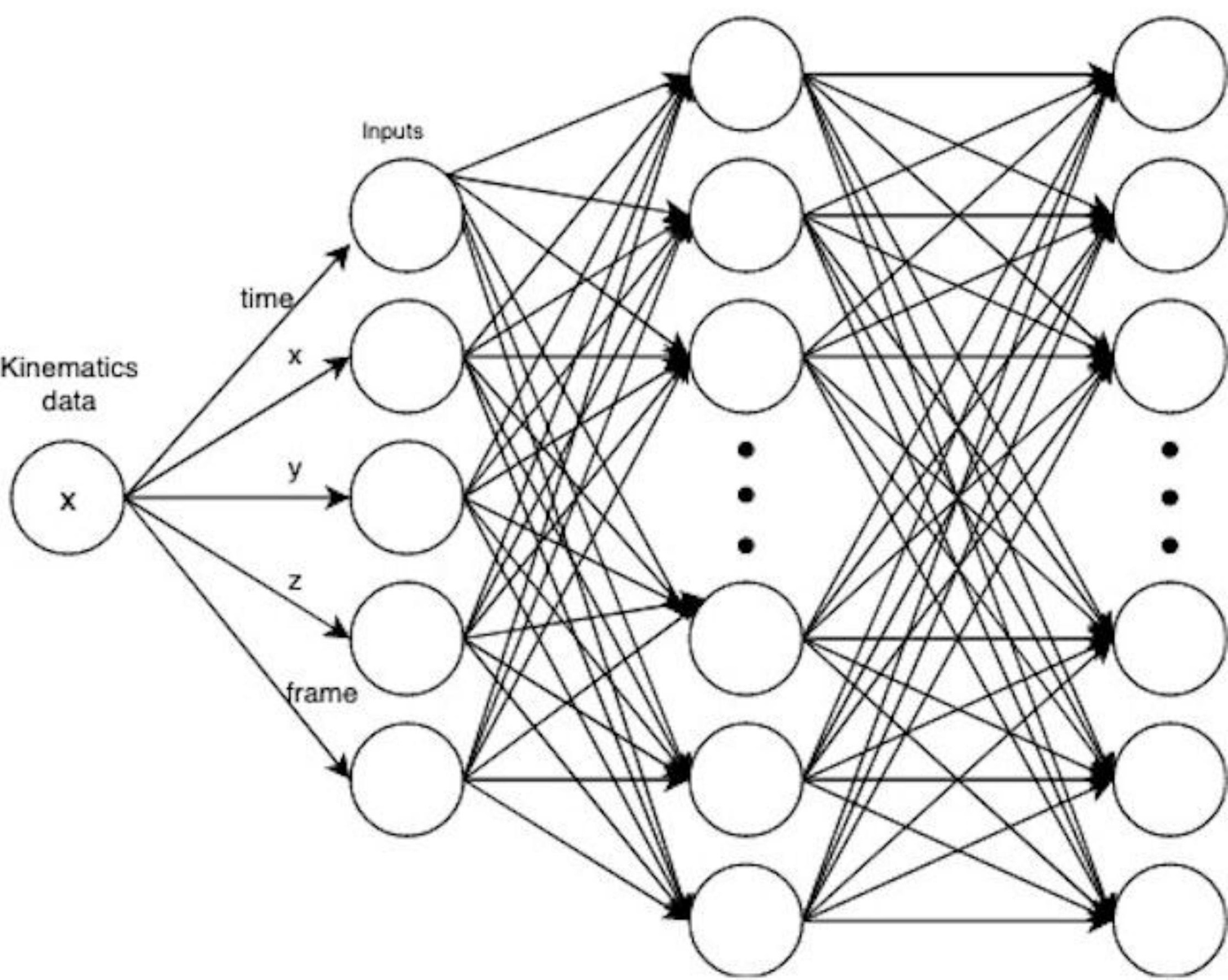




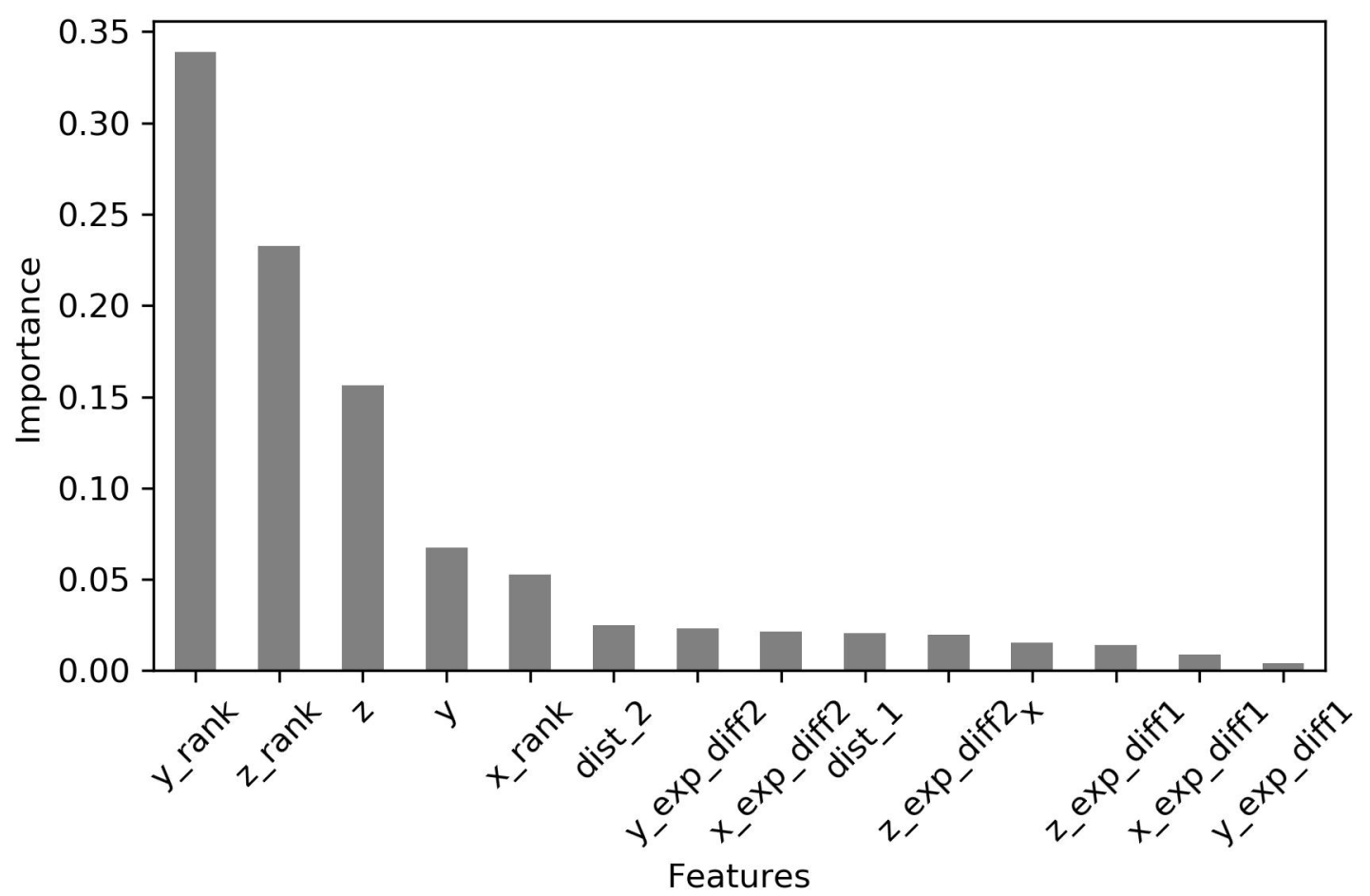




\section{Confusion Matrix from XGboost}

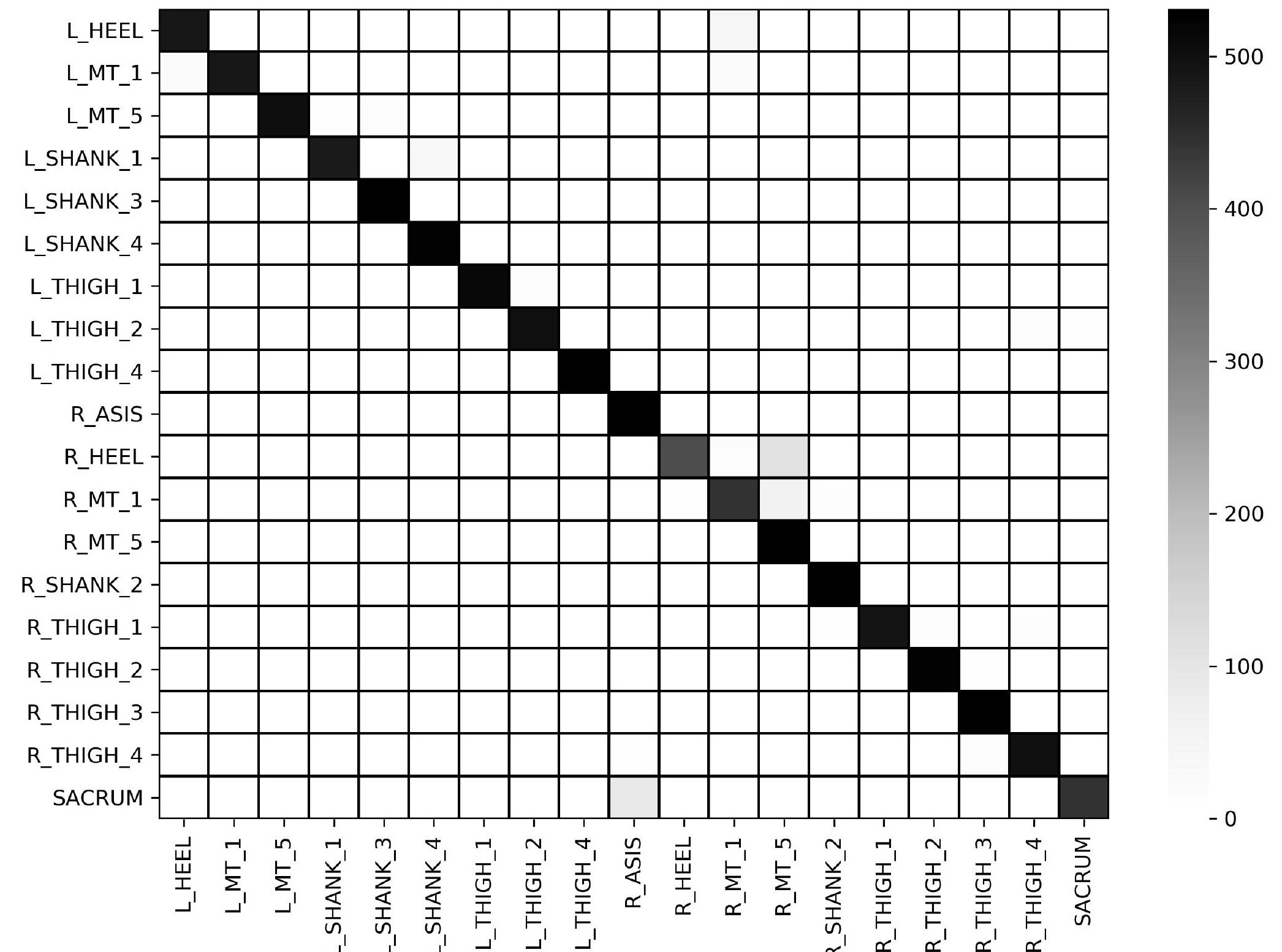

